02,13

\title{
Резонансные ступени тока в джозефсоновских структурах с прослойкой из материала, обладающего сильным спин-орбитальным взаимодействием
}

\author{
(C) К.И. Константинян ${ }^{1}$, Г.А. Овсянников ${ }^{1}$, А.М. Петржик ${ }^{1}$, А.В. Шадрин ${ }^{1,2}$, \\ Ю.В. Кислинский ${ }^{1}$, G. Cristiani ${ }^{3}$, G. Logvenov ${ }^{3}$
}

${ }^{1}$ Институт радиотехники и электроники им. В.А. Котельникова РАН, Москва, Россия

${ }^{2}$ Московский фризико-технический институт,

Долгопрудный, Россия

${ }^{3}$ Max Planck Institute for Solid State Research,

Stuttgart, Germany

E-mail: karen@hitech.cplire.ru

Поступила в Редакцию 26 марта 2020 г.

В окончательной редакции 26 марта 2020 г.

Принята к публикации 2 апреля 2020 г.

\begin{abstract}
Исследованы СВЧ- и магнитные параметры джозефсоновских меза-структур $\mathrm{Nb} / \mathrm{Au} / \mathrm{Sr}_{2} \mathrm{IrO}_{4} / \mathrm{YBa}_{2} \mathrm{Cu}_{3} \mathrm{O}_{x}$ с прослойкой из $\mathrm{Sr}_{2} \mathrm{IrO}_{4}$ - материала, являющегося моттовским антиферромагнитным изолятором с высокой энергией спин-орбитального взаимодействия, $E_{\mathrm{SO}} \sim 0.4 \mathrm{eV}$. Под воздействием монохроматического электромагнитного облучения возникали осциллирующие с мощностью облучения ступени Шапиро, подтверждающие джозефсоновские свойства структур. В присутствии слабого магнитного поля $H<15 \mathrm{Oe}$ на вольт-амперных характеристиках наблюдаются резонансные ступени тока при напряжениях $V_{n}$, обратно пропорциональных размеру структур в плане $L$. При смене полярности электрического тока $I$ наблюдалась асимметрия положения резонансных ступеней тока. При задании магнитного поля $H$ значения напряжений $V_{n}$ не изменялись, а амплитуды резонансных ступеней тока изменялись немонотонно.
\end{abstract}

Ключевые слова: меза-гетероструктура, спин-орбитальное взаимодействие, иридат стронция, ступени Фиске.

DOI: 10.21883/FTT.2020.09.49758.03H

\section{1. Введение}

В последнее время наблюдается повышенный интерес к сверхпроводниковым структурам с сильным спинорбитальным взаимодействием (СОВ), что обусловлено, в частности, возможностью возникновения в них спинтриплетного спаривания $[1,2]$ и перспективой реализации джозефсоновских структур с нарушенной инвариантностью при обращении времени $[3,4]$. Экспериментальное наблюдение эффекта Джозефсона в мезаструктурах $\mathrm{Nb} / \mathrm{Au} / \mathrm{Sr}_{2} \mathrm{IrO}_{4} / \mathrm{YBa}_{2} \mathrm{Cu}_{3} \mathrm{O}_{x}$ с барьерной прослойкой из $\mathrm{Sr}_{2} \mathrm{IrO}_{4}$ - материала, являющегося скошенным (canted) моттовским антиферромагнитным изолятором с высокой энергией $\mathrm{COB} E_{\mathrm{SO}} \sim 0.4 \mathrm{eV}$ и слабым ферромагнетизмом $\sim 0.04 \mu_{\mathrm{B}}$ на атом Ir сообщалось в работе [5]. Особенностью указанных структур являлось одновременное сосуществование сверхпроводимости тока в меза-структурах, пика проводимости при нулевом смещении (ZBCP) и рост проводимости при напряжениях $V>5-10 \mathrm{mV}$.

Известно, что под воздействием даже слабого магнитного поля на вольт-амперной характеристике (BAX) туннельного SIS-перехода $(S-$ сверхпроводниковые электроды, $I-$ немагнитный изолятор) возникают резонансные ступени Фиске [7,8] при напряжениях $V_{n}=n \Phi_{0} c^{\prime} / 2 L$, где $n-$ номер ступени, $\Phi_{0}-$ квант магнитного потока, $c^{\prime}=c(t / \varepsilon \Lambda)^{1 / 2}-$ скорость Свайхарта [9], $c-$ скорость света в вакууме, $L-$ ширина перехода, $t$ - толщина изоляторного слоя передающей линии с диэлектрической проницаемостью $\varepsilon, \Lambda$ глубина проникновения магнитного поля в прослойку и сверхпроводники. В случае сверхпроводящего туннельного перехода с изолятором, обладающего магнитными свойствами, величина глубины проникновения видоизменяется

$$
\Lambda=\mu t+\lambda_{L 1} \operatorname{cth}\left(d_{1} / 2 \lambda_{L 1}\right)+\lambda_{L 2} \operatorname{cth}\left(d_{2} / 2 \lambda_{L 2}\right),
$$

где $\mu$ - магнитная проницаемость, $d_{i}$ и $\lambda_{L i}(i=1,2)-$ толщины сверхпроводящих пленок и их лондоновские глубины проникновения магнитного поля, соответственно.

Влияние магнетизма барьерного слоя на динамику распространения электромагнитных колебаний в сверхпроводниковых контактах было рассмотрено теоретически для SIFS- и SFIFS-структур [10] и для $\mathrm{SI}_{\mathrm{F}} \mathrm{S}$ [11], здесь $\mathrm{F}$ - ферромагнетик, $I_{\mathrm{F}}-$ ферромагнитный изолятор. Однако в экспериментальных работах $[12,13]$ на SIFS-структурах отклонения от теории [7], предсказан- 
ные в работах [10,11], не отмечались. В то же время наличие сильного COB в прослойке $I_{\mathrm{SO}}$ может изменить динамику распространения электромагнитных волн в $S I_{\mathrm{SO}} S$-структуре. В настоящей работе сообщается об экспериментальном исследовании резонансных ступеней тока в $S_{1} I_{\mathrm{SO}} S_{2}$-структурах $\mathrm{Nb} / \mathrm{Au} / \mathrm{Sr}_{2} \mathrm{IrO}_{4} / \mathrm{YBa}_{2} \mathrm{Cu}_{3} \mathrm{O}_{x}$ с барьерной прослойкой из $\mathrm{Sr}_{2} \mathrm{IrO}_{4}$, материала с высокой энергией СОВ.

\section{2. Результаты и обсуждение}

Технология изготовления и результаты измерения электрофизических параметров сверхпроводниковых меза-структур (СМС) сообщались в работах $[6,14,15]$. СМС изготавливались из эпитаксиальных гетероструктур $\mathrm{Sr}_{2} \mathrm{IrO}_{4} / \mathrm{YBa}_{2} \mathrm{Cu}_{3} \mathrm{O}_{x}$ с толщиной пленки $\mathrm{YBa}_{2} \mathrm{Cu}_{3} \mathrm{O}_{x} \sim 100 \mathrm{~nm}$ дополнительным напылением пленок $\mathrm{Nb}$ и $\mathrm{Au}$, а также изолятора $\mathrm{SiO}_{2}$. Топология меза-структур микронных размеров формировалась с помощью фотолитографии и ионно-плазменного и ионно-лучевого травлений. В данной работе обсуждаются результаты экспериментального исследования СМС $\mathrm{Nb} / \mathrm{Au} / \mathrm{Sr}_{2} \mathrm{IrO}_{4} / \mathrm{YBa}_{2} \mathrm{Cu}_{3} \mathrm{O}_{x}$, у которых прослойка $\mathrm{Sr}_{2} \mathrm{IrO}_{4}$ имела толщину $t=5 \mathrm{~nm}$, а размеры $L$ в плане изменялись от 20 до $50 \mu \mathrm{m}$.

На рис. 1 приведена магнитнополевая зависимость критического тока $I_{C}(H)$, представленная для обеих полярностей задаваемого тока I через СМС. Отметим, магнитное поле задавалось соленоидом, ток через который изменялся от $I_{H}=0$, затем до положительного значения $I_{H}$ (поле $\left.H_{+}\right)$и обратно до отрицательного значения $\left(H_{-}\right)$. На рис. 1 приведена зависимость, снятая при изменении поля от $H_{+}=13.2 \mathrm{Oe}$ до $H_{-}=-13.7 \mathrm{Oe}$. Сoленоид и СМС находились внутри экрана из многослойного аморфного пермаллоя, понижающего поле Земли примерно на порядок. Величины малых значений критического тока $I_{C}$ и замытых флуктуациями резонансных ступеней $I_{n}$ определялись по методике, приведенной в работе [6]. Семейства $\mathrm{BAX}$ снимались в режиме задания тока в последовательности от $0 \rightarrow I_{+} \rightarrow I_{-} \rightarrow 0$. Здесь $I_{+}$и $I_{-}-$крайние значения измерительных токов с индексом, указывающим полярность. Аналогичное обозначение использовалось для значений критического тока с разной полярностью: $I_{C+}$ и $I_{C-}$. Из рис. 1 видно, что на осциллирующих зависимостях $I_{C+}(H)$ и $I_{C-}(H)$ в диапазоне $H$ от -15 до -7 Ое и при $H>10$ Ое имеются нулевые минимумы, что свидетельствует об отсутствии закороток. Расчетное значение первого минимума по теоретической фраунгоферовой зависимости $I_{C}(H)$ дает близкое к эксперименту значение $H_{1}=\Phi_{0} / \mu_{0} \Lambda L \approx 4$ Ое при $\lambda_{L 1}=150 \mathrm{~nm}$ для $\mathrm{YBa}_{2} \mathrm{Cu}_{3} \mathrm{O}_{x}$ и $\lambda_{L 2}=90 \mathrm{~nm}$ для $\mathrm{Nb}$, хотя форма экспериментальной зависимости $I_{C}(H)$ для $H>0$ заметно отличается от фраунгоферовой. Наблюдается отличие $I_{C+}(H)$ и $I_{C-}(H)$ как по направлению магнитного поля, $H>0$ и $H<0$, так и по полярности задания измерительного тока I через СМС. Зависимость

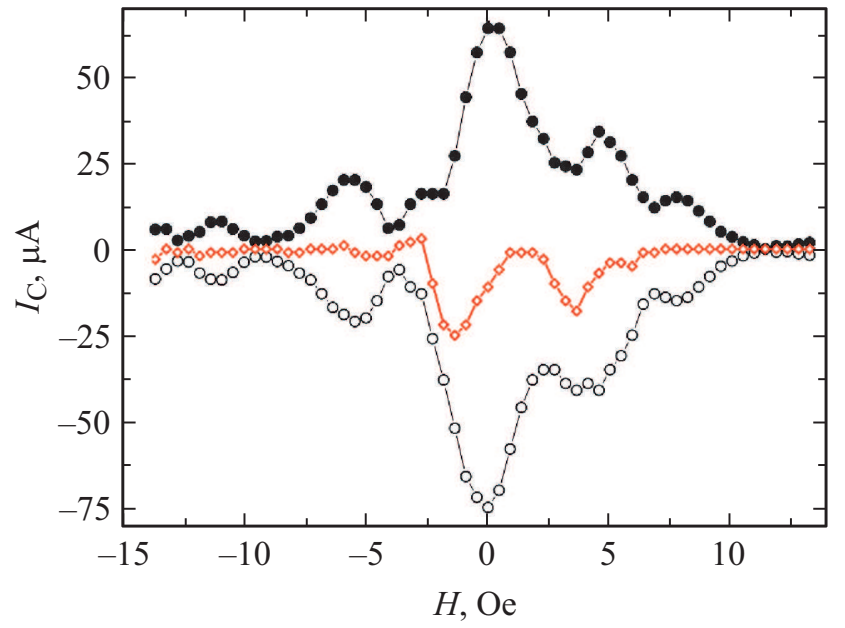

Рис. 1. Зависимости $I_{C}(H)$ для СМС с $L=40 \mu \mathrm{m}$. Темные кружки соответствуют положительной полярности задаваемого тока $I$, незаполненные - светлые. Магнитная зависимость разности значений критических токов при положительном и отрицательном задании электрического смещения показана ромбами.

$I_{C+}(H)$ vs $I_{C-}(H)$, наблюдаемая в узком интервале полей $H$, также приведена на рис. 1. Заметим, случай „широкого“ перехода $L>4 \lambda_{J}$, где $\lambda_{J}=\left(\Phi_{0} / \mu_{0} \Lambda j_{C}\right)^{1 / 2}-$ джозефсоновская глубина проникновения магнитного поля $\left(j_{C}=I_{C} / L^{2}-\right.$ плотность критического тока) не объясняет асимметрию и отличие критических токов $I_{C+}$ и $I_{C-}$, поскольку $\lambda_{J}=170 \mu \mathrm{m}$ и имеем, напротив, обратное неравенство $\lambda_{J}>4 L$.

Были проведены также измерения BAX и зависимостей дифференциального сопротивления $R_{D}(V)$ под воздействием электромагнитного облучения на частоте $f_{e}=50 \mathrm{GHz}$ при различных значениях мощности воздействия $P$ (см. рис. 2). Видно, что эквидистантность напряжений $V_{N}=N \Phi_{0} f_{e}$ ступеней Шапиро выполняется с высокой точностью для обеих полярностей напряжения $V$, как показано на рисунке для $N=1,2,3$. В то же время амплитуды ступеней Шапиро, судя по глубине минимумов $R_{D}$, нормированных на $R_{N}$, для разнополярных значений $V$ отличаются. Такая асимметрия может быть обусловлена отличием спин-поляризованных компонент тока через СМС, что требует отдельного исследования. Важно отметить осциллирующие с мощностью электромагнитного облучения амплитуды $I_{C}$ и ступеней Шапиро, также свидетельствующие об отсутствии закороток. На рис. 2 приведена также зависимость $R_{D}(V)$, снятая без СВЧ-воздействия (кривая $a$ ). Видно, что уже слабое воздействие с 30-dB затуханием мощности $P$ практически нивелирует резонансные особенности тока, а ступени Шапиро с номером до $N=2$ хорошо регистрируются.

Положение резонансных ступеней тока по напряжению определялись по минимумам дифференциального сопротивления $R_{D}=d V / d I$ СМС при воздействии магнитного поля $H$. На рис. 3 показана зависимость диф- 
ференциального сопротивления $R_{D}$ от напряжения при воздействии магнитного поля $H=-1.3 \mathrm{Oe}$. Цифрами на рис. 3 указаны номера минимумов $R_{D}$. При этом значении магнитного поля хорошо видны минимумы $R_{D}$ до $n=3$. Соответствующая зависимость $R_{D}(I) \mathrm{CMC}$, указывающая на наличие критического тока, приведена на вставке к рис. 3.

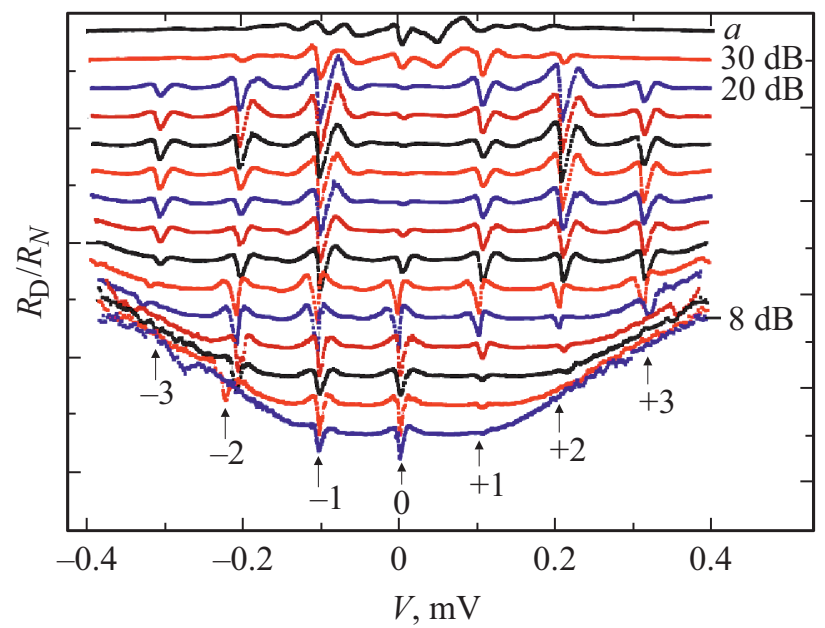

Рис. 2. Зависимости нормированных к $R_{N}$ значений дифференциального сопротивления $R_{D}$ от напряжения $V$, снятых под воздействием электромагнитного облучения на $f=50.09 \mathrm{GHz}$ для МС с $L=40 \mu \mathrm{m}$. Кривые сдвинуты по оси ординат. Цифрами указаны величины вносимого аттенюатором затухания мощности воздействия $P: 30 \mathrm{~dB}$, затем с шагом по $1 \mathrm{~dB}$ от 20 до $8 \mathrm{~dB}$. Кривая $a$ снята без СВЧ-воздействия. Цифрами указаны номера ступеней Шапиро $N, 0$ соответствует критическому току.

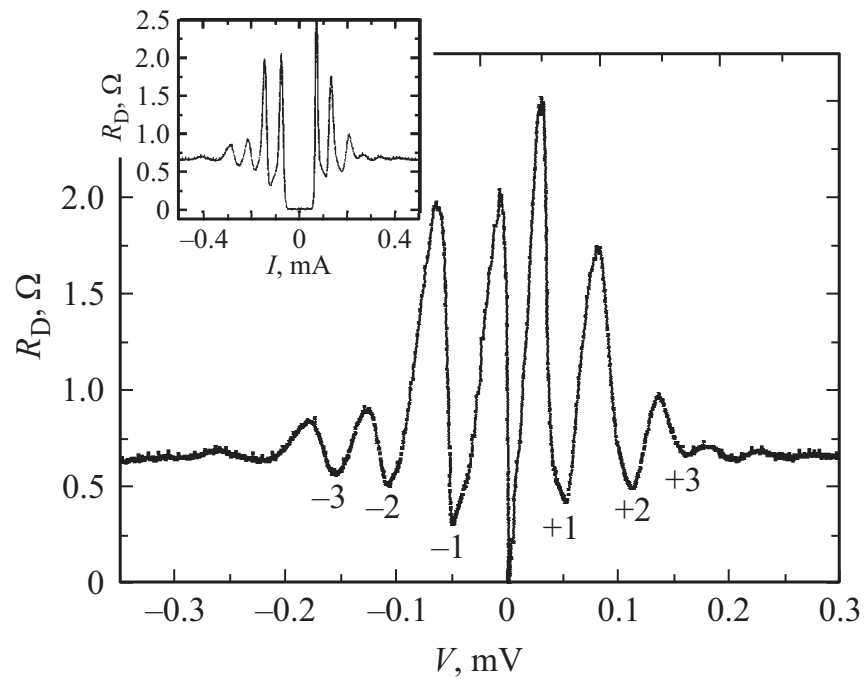

Рис. 3. Зависимость дифференциального сопротивления $R_{D}$ CMC с $L=40 \mu \mathrm{m}$ от напряжения $V$ при напряженности магнитного поля $H=-1.3 \mathrm{Oe}$, соответствующая подавлению на $30 \%$ критического тока $I_{C}$ на ветви ВАХ, снятой при отрицательном смещении $(V<0)$ при $T=4.2 \mathrm{~K}$. Цифрами указаны номера $n$ минимумов $R_{D}$. На вставке показана зависимость $R_{D}$ от тока $I$.

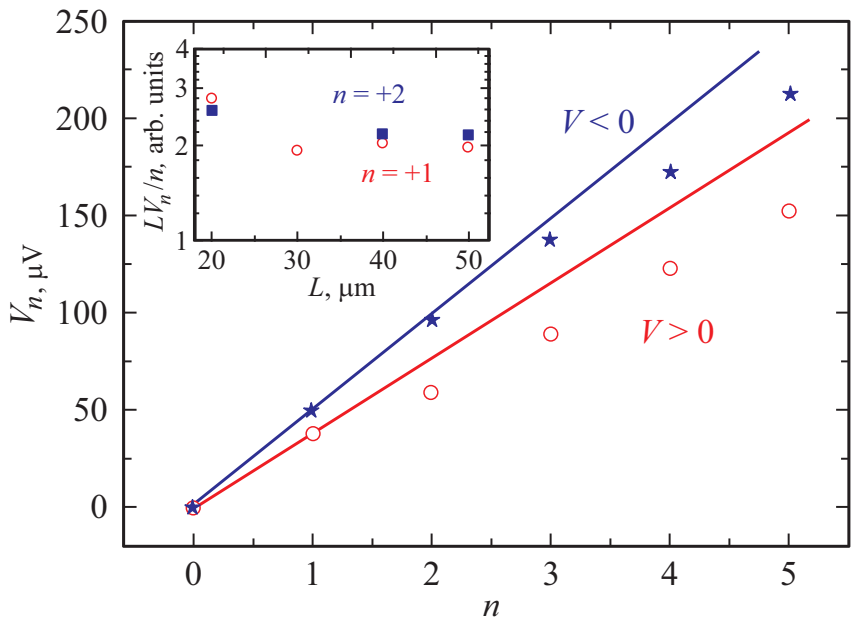

Рис. 4. Значения напряжений резонансных ступеней $V_{n}$ на CMC с $L=40 \mu \mathrm{m}$ при положительных и отрицательных напряжениях смещения. Прямые линии - линейные зависимости $V_{n}(n)$, отвечающие условию эквидистантности напряжений $V_{n}$ относительно $V_{1}$ для $n=+1(V>0)$ и $n=-1(V<0)$. На вставке показана зависимость скорости Свайхарта (параметр $\left.L V_{n} / n\right)$ от $L$ для четырех СМС на одном чипе для $n=+1$ и $n=+2$.

На рис. 4 приведены значения напряжений $V_{n}$, соответствующие минимумам $R_{D}$ для $n=1-5$ для той же СМС. Видно, что отсутствует эквидистантность положений резонансных ступеней по напряжению. Кроме того, наблюдается асимметрия по напряжению $V$ на $\mathrm{CMC}$; так, значения напряжения особенностей (минимумов $R_{D}$ ) для $n=+1$ и $n=-1$, снятых при различных полярностях напряжения, отличаются более чем на $10 \mu \mathrm{V}$. Отметим, что ошибка при определении особенностей определяется влиянием шумов и не превышает $\pm 0.25 \mu \mathrm{V}$.

На вставке к рис. 4 показаны значения $V_{n} L / n$ с номерами резонансных ступеней $n=1$ и 2 для четырех CMC на одном чипе, скорости Свайхарта которых должны быть одинаковы из-за постоянства $t / \varepsilon \Lambda$. Такая зависимость $V_{n}$ от ширины $L$ соответствует возникновению ступенек Фиске [7]. Видно, что отклонение параметра $V_{n} L / n$, характеризующего величину $c^{\prime}$, от среднего значения для СМC с $L=30,40$ и $50 \mu \mathrm{m}$ оказалось порядка $5 \%$ и несколько больше для СМС с $L=20 \mu \mathrm{m}$. Небольшой сдвиг напряжений ступеней Фиске с высокими номерами $n$ отмечался в [13], что объяснялось влиянием окружающей среды с диэлектрическими свойствами, отличными от материала туннельного барьера [16]. Однако в нашем случае отклонение начинается уже с $n=1$. Учитывая высокое значение $\varepsilon \sim 45$, измеренное для монокристаллического $\mathrm{Sr}_{2} \mathrm{IrO}_{4}$ [17], влияние диэлектрического окружения $\mathrm{SiO}_{2}$ в нашем случае может сказаться только при $n \gg 1$.

На рис. 5 приведены амплитуды ступеней Фиске для $n=+1$ и $n=-1$, снятых при магнитном поле $H<0$, где форма $I_{C}(H)$ ближе следует теоретической фраунго- 
феровой зависимости, приведенной на рис. 5, $a$. Подгоночными параметрами для фраунгоферовой зависимости использовались значения первых двух минимумов $I_{C}(H)$ и среднее значение амплитуды $\left(I_{C+}+I_{C-}\right) / 2$. Видно наличие ступеней даже при $H=0$, что вызвано, скорее всего, асимметрией сверхпроводящего тока $I_{C+} \neq I_{C-}$ (см. рис. 1). На рис. 5, $b$ приведена теоретическая зависимость [8] от магнитного поля амплитуды ступени Фиске $I_{-1}$ с $n=-1$ при напряжении $V_{-1}$. В качестве подгоночных параметров по полю $H$ для теоретической функции $I_{-1}(H)$ использовались значения магнитного поля первых двух максимумов $I_{-1}(H)$ со сдвигом по $H$ на 1 Ое (или на $\left.\Phi_{0} / 6\right)$, что приводит также к сдвигу нуля $I_{1}(H)$ при $H=0$ по сравнению с теорией [8]. Отметим осциллирующий характер магнитно-полевых зависимостей $I_{+1}$ и $I_{-1}$ и относительно высокие амплитуды вторичных максимумов по сравнению с теоретической. На рис. 6 показаны все зарегистрирован-
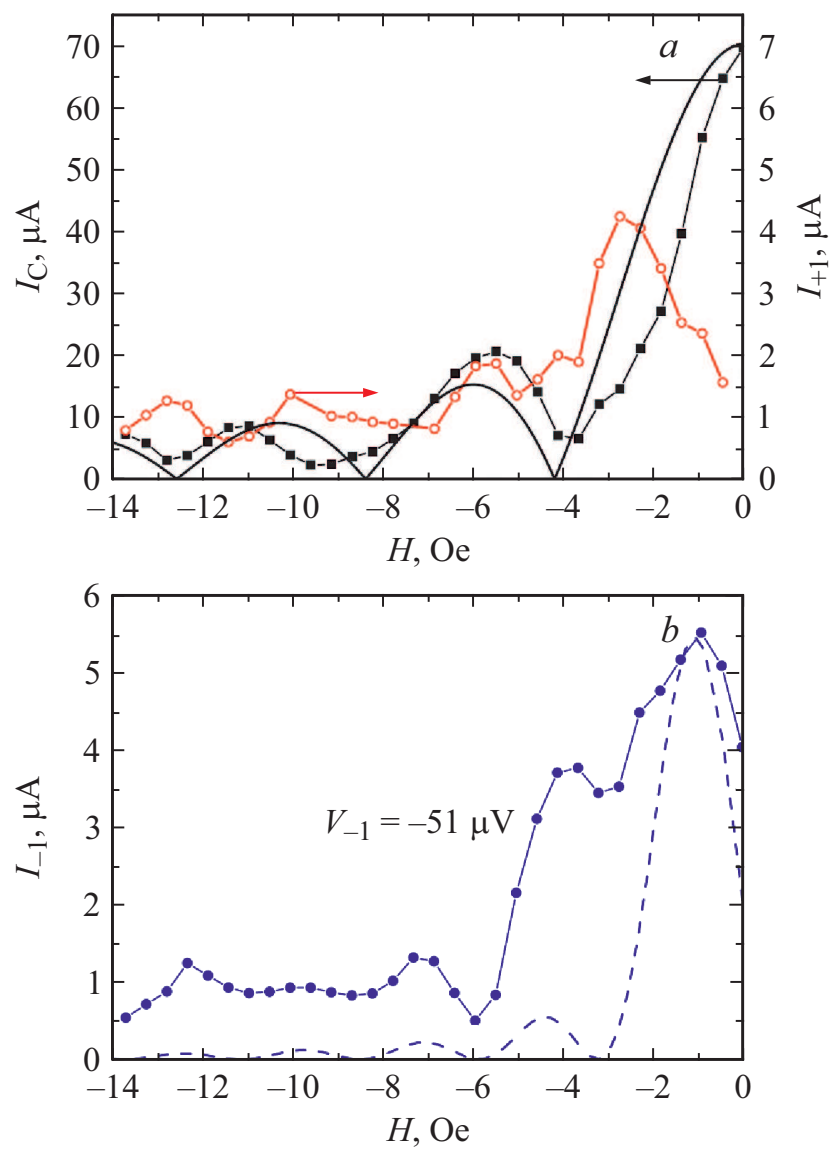

Pис. 5. a) Зависимости от магнитного поля среднего значения критического тока $I_{C}=\left(I_{C+}+I_{C-}\right) / 2$ (квадраты) и амплитуды ступеней Фиске $I_{+1}(H)$ для $n=+1$ при $V=+39 \mu \mathrm{V}$. Теоретическая фраунгоферова зависимость $I_{C}(H)$ показана сплошной линией. $b$ ) Зависимости от магнитного поля амплитуды ступеней Фиске $I_{-1}(H)$ для $n=-1$ при $V=-51 \mu \mathrm{V}$. Теоретическая зависимость $I_{-1}(H)$ показана штриховой линией. Максимальные значения теоретических значений $I_{C}$ и $I_{-1}$ совмещены с экспериментальными.

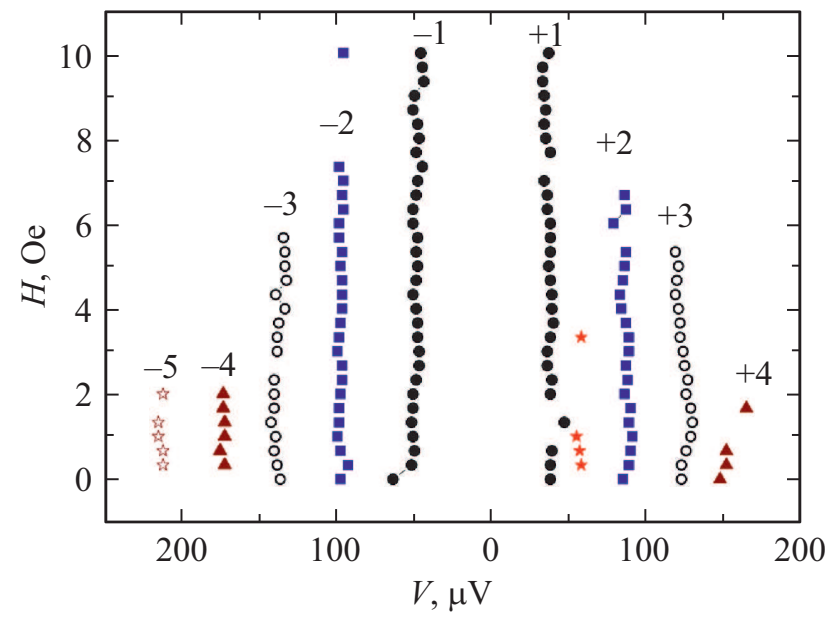

Рис. 6. Ступени Фиске на плоскости $H-V$. Цифрами указаны номера $n$ ступеней.

ные резонансные токовые ступени Фиске с достаточно хорошо идентифицируемыми номерами $n$. Также видно, что положения ступеней Фиске по напряжению $V_{n}$ устойчивы к изменению магнитного поля. Отклонение от эквидистантности и отличие $V_{n}$ при смене знака $n$ могут быть вызваны влиянием на спектр джозефсоновских плазменных волн колебаний локальной намагниченности антиферромагнитной прослойки с сильным спин-орбитальным взаимодействием. Для аномального эффекта Джозефсона со сдвигом фазы на $\varphi_{0}$ требуется реализация условий, например, расщепления спиновых зон и наличия СОВ $[18,19]$. В нашем эксперименте уровень приложенного магнитного поля много меньше зеемановского расщепления, но на характеристики СМС могут оказать значительное влияние магнон-плазменное взаимодействие волн, рассмотренное теоретически для ферромагнитного случая $[10,11]$. Однако вопрос взаимодействия плазменных и спиновых волн в антиферромагнетике с сильным СОВ пока остается открытым.

\section{3. Заключение}

В сверхпроводящих меза-структурах $\mathrm{Nb} / \mathrm{Au} / \mathrm{Sr}_{2} \mathrm{IrO}_{4} /$ $\mathrm{YBa}_{2} \mathrm{Cu}_{3} \mathrm{O}_{x}$ с прослойкой из $\mathrm{Sr}_{2} \mathrm{IrO}_{4}$ толщиной $5 \mathrm{~nm}$, эпитаксиально выращенной на пленке $\mathrm{YBa}_{2} \mathrm{Cu}_{3} \mathrm{O}_{x}$, наблюдаются резонансные ступени тока и эффект Джозефсона. Наблюдалось неравенство амплитуд критического тока $I_{C+} \neq I_{C-}$ при смене полярности задания электрического тока через структуру. Под воздействием электромагнитного облучения миллиметрового диапазона волн возникают осциллирующие с мощностью ступени Шапиро, указывающие на отсутствие закороток, как и нулевые минимумы на зависимости $I_{C}(H)$. Отклонение от эквидистантности осциллирующих с полем- $H$ ступеней Фиске и асимметрия зависимости $I_{C}(H)$ вызваны, скорее всего, влиянием сильного спин-орбитального взаимодействия материала барьерной прослойки $\mathrm{Sr}_{2} \mathrm{IrO}_{4}$, 
известным как антиферромагнитный изолятор с высоким значением диэлектрической проницаемости.

\section{Благодарности}

Авторы благодарны А.В. Зайцеву и В.П. Кошельцу за полезные обсуждения.

\section{Финансирование работы}

Работа выполнена в рамках государственного задания и частично поддержана РФФИ (проект 19-07-00274).

\section{Конфликт интересов}

Авторы заявляют, что у них нет конфликта интересов.

\section{Список литературы}

[1] C.R. Reeg, D.L. Maslov. Phys. Rev. B 92, 134512 (2015).

[2] I.V. Bobkova, A.M. Bobkov. Phys. Rev. B 95, 184518 (2017).

[3] O.V. Dimitrova, M.V. Feigel'man. ЖЭТФ 129, 4, 742 (2006).

[4] A. Buzdin. Phys. Rev. Lett. 101, 107005 (2008).

[5] G. Cao, P. Schlottmann. Rep. Prog. Phys. 81, 042502 (2018).

[6] A.M. Petrzhik, K.Y. Constantinian, G.A. Ovsyannikov, A.V. Zaitsev, A.V. Shadrin, A.S. Grishin, Yu.V. Kislinski, G. Cristiani, G. Logvenov. Phys. Rev. B 100, 024501 (2019).

[7] D.D. Coon, M.D. Fiske. Phys. Rev. 138, A744 (1965).

[8] И.О. Кулик. Письма в ЖЭТФ 2, 134 (1965).

[9] J.C. Swihart. J. Appl. Phys. 32, 461 (1961).

[10] S. Mai, E. Kandelaki, A.F. Volkov, K.B. Efetov. Phys. Rev. B 84, 144519 (2011).

[11] S.-I. Hikino, M. Mori, S. Takahashi, S. Maekawa. J. Phys. Soc. Jpn. 80, 074707 (2011).

[12] G. Wild, C. Probst, A. Marx, R. Gross. Eur. Phys. J. B 78, 509 (2010).

[13] J. Pfeiffer, M. Kemmler. Phys. Rev. B 77, 214506 (2008).

[14] Ю.В. Кислинский, Г.А. Овсянников, А.М. Петржик, К.И. Константинян, Н.В. Андреев, Т.А. Свиридова. ФТТ 57, 2446 (2015).

[15] Г.А. Овсянников, А.С. Гришин, К.И. Константинян, А.В. Шадрин, А.М. Петржик, Ю.В. Кислинский, G. Cristiani, G. Logvenov. ФTT 60, 11, 2125 (2018).

[16] R. Monaco, G. Costabile, N. Martuccielle. J. Appl. Phys. 77, 5, 2073 (1995)

[17] S. Chikara, O. Korneta, W.P. Crummett, L.E. DeLong, P. Schlottmann, G. Cao. Phys. Rev. B 80, 14, 140407R (2009).

[18] M.A. Silaev, I.V. Tokatly, F.S. Bergeret. Phys. Rev. B 95, 184508 (2017).

[19] S. Calder. Phys. Rev. B 98, 220402(R) (2018).

Редактор Е.В. Толстякова 